\title{
Nonlocal fluctuations and control of dimer entanglement dynamics
}

\author{
Cristian E. Susa and John H. Reina* \\ Departamento de Física, Universidad del Valle, A.A. 25360, Cali, Colombia
}

(Dated: October 31, 2018)

\begin{abstract}
We report on the dissipative dynamics of an entangled, bipartite interacting system. We show how to induce and control the so-called early stage disentanglement (and the 'delayed' entanglement generation) dynamics by means of a driving laser field. We demonstrate that some of the features currently associated with pure non-Markovian effects in such entanglement behavior can actually take place in Markovian environments if background noise QED fluctuations are considered. We illustrate this for the case of a dimer interacting molecular system for which emission rates, interaction strength, and radiative corrections have been previously measured. We also show that even in the absence of collective decay mechanisms and qubit-qubit interactions, the entanglement still exhibits collapse-revival behavior. Our results indicate that zero point energy fluctuations should be taken into account when formulating precise entanglement dynamics statements.
\end{abstract}

PACS numbers: 03.65.Ud, 03.67.Mn, 42.50.Fx, 42.50.Lc, 33.50.Dq

\section{INTRODUCTION}

Entanglement is arguably the most striking feature of quantum phenomena [1, and is a crucial physical resource to quantum computing [2], and quantum communication via protocols such as teleportation [3] and cryptography 4. Works on entanglement dynamics have recently shown that some initial bipartite entangled states can decay to zero in a finite time much shorter than that of their spontaneous emission 5-11. This phenomenonearly stage disentanglement (ESD)- is a quantum feature that signals an unusual dissipative dynamics of purely non-local quantum correlations [1, 3]. It has also recently been shown that, in specific scenarios, it is possible to produce a delayed creation of entanglement, the socalled entanglement sudden birth (ESB) [12, 13]. These entanglement features have been explored in both the Markovian 14 and Non-Markovian [15] regimes in several different contexts [11 16]. Even more recently, there have been reports on the quantification of non-classical correlations of bipartite qubit systems in interaction with non-Markovian reservoirs [17 by means of the quantum discord [18] dynamics, in contrast to that of entanglement evolution [17.

In this work, we show how the ESD and ESB phenomena can be externally coherently controlled with a laser field. We also show that the entanglement dynamics is permanently affected by the background noise due to nonlocal vacuum fluctuations, a fact that permanently affects the ESD dynamical profile. Our results give a practical prescription, within the Born-Markov formalism, for the identification and control of ESD, ESB, and collapserevival phenomena. This shows that previously identified entanglement dynamics associated to non-Markovian behavior [15, can actually take place within a Markovian dynamics description.

\footnotetext{
*Electronic address: jhreina@univalle.edu.co
}

Most of the interacting two-qubit systems can be described by a Hamiltonian of the type [19] $\hat{H}_{S}=\hat{H}_{0}+\hat{H}_{12}$, where $\hat{H}_{0}$ denotes the free particle term, and $\hat{H}_{12}=$ $h\left(J_{x} \sigma_{x}^{(1)} \otimes \sigma_{x}^{(2)}+J_{y} \sigma_{y}^{(1)} \otimes \sigma_{y}^{(2)}+J_{z} \sigma_{z}^{(1)} \otimes \sigma_{z}^{(2)}\right)$, written in terms of the Pauli matrices, denotes a generic, anisotropic qubit-qubit interaction, and $h$ is Planck's constant. In many physical processes, such as energy transfer in biomolecular systems [16, 20, or interacting artificial atoms [21, to name but a few, $J_{x}=J_{y} \equiv J_{x y}$, and the strength ratio $J \equiv J_{x y} / J_{z}$ sets the type of interaction experienced by the coupled qubits, and defines the type of entanglement that can be 'naturally' 19] generated in the bipartite system.

In this article, we consider the case of dipole-dipole (d-d) interacting single molecules of transition frequencies $\nu_{i}$ (molecule $i$ ) in interaction with the quantized radiation field, and externally driven by a coherent laser field [22, 23]. We denote by $\left|0_{i}\right\rangle$, and $\left|1_{i}\right\rangle, i=1,2$, the ground and excited state of molecule $i$, respectively, and hence $\hat{H}_{0}=-\frac{h}{2} \nu_{1} \sigma_{z}^{(1)}-\frac{h}{2} \nu_{2} \sigma_{z}^{(2)}$. The two two-level molecules are separated by the vector $\mathbf{r}_{12}$ and are characterized by transition dipole moments $\hat{\boldsymbol{\mu}}_{i} \equiv\left\langle 0_{i}\left|\mathbf{D}_{i}\right| 1_{i}\right\rangle$, with dipole operators $\mathbf{D}_{i}$, and spontaneous emission rates $\Gamma_{i}$. The system interaction Hamiltonian can be written in the computational basis of direct product states $|i\rangle \otimes|j\rangle$ $(i, j=0,1)$ as $\hat{H}_{S}=\hat{H}_{0}+\hat{H}_{12}$, where $\hat{H}_{12}$ is set by the dipole coupled molecules of interaction energy $h V_{12}$ $\left(J_{x}=J_{y} \equiv V_{12}, J_{z} \equiv 0\right)$.

\section{DIMER DISSIPATIVE DYNAMICS}

Evidence of the molecules dipolar coupling, and the generation of sub- and super-radiant states, as well as an effective shift of the doubly excited state, have been experimentally reported in 22]. The latter has been accounted for in $\hat{H}_{12}$ as a phenomenological, effective $\Delta_{e^{-}}$ shift of the doubly-excited state $|11\rangle \equiv\left|1_{1}\right\rangle \otimes\left|1_{2}\right\rangle$, due to the vacuum fluctuations 23 . The dimer is externally 
controlled by a coherent driving field which acts on each of the molecules with a coupling amplitude $h \ell_{i}=-\boldsymbol{\mu}_{i} \cdot \boldsymbol{E}_{i}$, and a frequency $\omega_{L}$. The light-matter interaction Hamiltonian $\hat{H}_{L}=h \ell^{(i)}\left(\sigma_{-}^{(i)} e^{i \omega_{L} t}+\sigma_{+}^{(i)} e^{-i \omega_{L} t}\right)$, where $\boldsymbol{\mu}_{i}$ is the $i$-th transition dipole moment and $\boldsymbol{E}_{i}$ is the amplitude of the coherent driving acting on molecule $i$ (at $\boldsymbol{r}_{i}$ ).

The dimer dissipative dynamics is appropriately described within the Born-Markov formalism, with $\hat{H}=$ $\hat{H}_{S}+\hat{H}_{L}$, by the quantum master equation

$$
\hat{\dot{\rho}}=-\frac{i}{\hbar}[\hat{H}, \hat{\rho}]+L(\hat{\rho}),
$$

with dissipative Lindblad super-operator given by [24]

$$
\begin{aligned}
L(\hat{\rho})= & -\frac{\Gamma_{1}}{2}\left(\hat{\rho} \sigma_{+}^{(1)} \sigma_{-}^{(1)}+\sigma_{+}^{(1)} \sigma_{-}^{(1)} \hat{\rho}-2 \sigma_{-}^{(1)} \hat{\rho} \sigma_{+}^{(1)}\right) \\
& -\frac{\Gamma_{2}}{2}\left(\hat{\rho} \sigma_{+}^{(2)} \sigma_{-}^{(2)}+\sigma_{+}^{(2)} \sigma_{-}^{(2)} \hat{\rho}-2 \sigma_{-}^{(2)} \hat{\rho} \sigma_{+}^{(2)}\right) \\
& -\frac{\Gamma_{12}}{2}\left(\hat{\rho} \sigma_{+}^{(1)} \sigma_{-}^{(2)}+\sigma_{+}^{(1)} \sigma_{-}^{(2)} \hat{\rho}-2 \sigma_{-}^{(1)} \hat{\rho} \sigma_{+}^{(2)}\right) \\
& -\frac{\Gamma_{21}}{2}\left(\hat{\rho} \sigma_{+}^{(2)} \sigma_{-}^{(1)}+\sigma_{+}^{(2)} \sigma_{-}^{(1)} \hat{\rho}-2 \sigma_{-}^{(2)} \hat{\rho} \sigma_{+}^{(1)}\right),
\end{aligned}
$$

where $\Gamma_{i i} \equiv \Gamma_{i}=n A_{12 ; i} / 2=n \omega_{i}^{3}\|\boldsymbol{\mu}\|^{2} /\left(3 \epsilon_{0} h c^{3}\right)$ are the spontaneous emission rates $\left(A_{12 ; i}, i=1,2\right.$ are the Einstein vacuum coefficients), $n$ is the refraction index of the dispersive medium in which the molecules are embedded (for example, a paraterphenyl crystal in [22]), and $\Gamma_{12}=\Gamma_{21}^{*}$ are the collective spontaneous emission rates, arising from the coupling between the molecules through the vacuum field $\left[24 ; \sigma_{+}^{(i)}=\left|1_{i}\right\rangle\left\langle 0_{i}\right|\right.$, and $\sigma_{-}^{(i)}=\left|0_{i}\right\rangle\left\langle 1_{i}\right|$ are the raising and lowering Pauli operators acting on molecule $i$.

In particular, in the near field approximation $\left(r_{12} \ll\right.$ $\left.\lambda_{L}\right)$ the $\mathrm{d}$-d interaction energy is given by $h V_{12}=$ $\frac{3 h \sqrt{\Gamma_{1} \Gamma_{2}}}{8 \pi z^{3}}\left[\hat{\boldsymbol{\mu}}_{1} \cdot \hat{\boldsymbol{\mu}}_{2}-3\left(\hat{\boldsymbol{\mu}}_{1} \cdot \hat{\mathbf{r}}_{12}\right)\left(\hat{\boldsymbol{\mu}}_{2} \cdot \hat{\mathbf{r}}_{12}\right)\right]$, and $\Gamma_{12}=$ $\sqrt{\Gamma_{1} \Gamma_{2}} \hat{\boldsymbol{\mu}}_{1} \cdot \hat{\boldsymbol{\mu}}_{2}$ : the maximum (minimum) interaction strengths $V_{12}$ are obtained for parallel (perpendicular) dipole moments [33. $z \equiv n k_{0} r_{12} ; \hat{\boldsymbol{\mu}}_{i}$, and $\hat{\mathbf{r}}_{12}$ are unit vectors along the transition dipole moments and along the relative separation between the molecules.

We stress that Eq. (1) is more general than that used in [11, 15] in the sense that it accounts for qubit-qubit coupling, coherent external driving, and nonlocal fluctuations due to radiative corrections [24.

\section{RESONANCE FLUORESCENCE}

To evaluate the system's physical observables, we perform the calculation of the occupation probabilities in the steady state. We compute this for several different experimental conditions realizable in the laboratory, as shown in Fig. 1. These involve weak and strong coherent laser driving (e.g., Fig. 1(c)), different dipole interaction strengths (Figs. 1(a), and 1(d)), and different dimer's resonance frequencies (Fig. 1(b)). In particular, we account for the fluorescence spectrum measured for the pair of coupled molecules reported in Ref. [22].

Our calculations are performed in the standard twoqubit computational basis $\{|00\rangle,|01\rangle,|10\rangle,|11\rangle\} \quad(|i j\rangle \equiv$ $\left.\left|i_{1}\right\rangle \otimes\left|j_{2}\right\rangle\right)$, and the corresponding dimer density matrix elements are denoted as $\rho_{i j, k l}$, with associated populations $\rho_{i j, i j}$ (density matrix elements $|i j\rangle\langle i j|$ ), $i, j=0,1$. The calculations here presented are amenable to experimental verification in a dimer molecular set-up where the task of optically resolving the single molecules by means of laser spectroscopy techniques is possible. This kind of experiment has been performed, for instance, by Hettich et al. 22, and the detection and measurement of coupled single terrylene molecules embedded in an organic (para-terphenyl) crystal (thickness of about $250 \mathrm{~nm}$ ) has been reported. There the detection was carried out by means of fluorescence excitation spectroscopy at a temperature $\sim 1.4 \mathrm{~K}$. Such a recorded fluorescence spectra, at high excitation laser intensity, exhibits the appearance of an 'additional' peak between those associated to the individual transition frequencies $\nu_{1}$, and $\nu_{2}$, which is the experimental evidence of the dipole-dipole coupling $V_{12}$ between the qubits.

The continuous curves shown in Fig. 1(a) are in perfect agreement with the experimental results reported in [22], for the same set of implemented physical parameters: difference in dipole transition frequencies $\Delta_{-}=2320$ $\mathrm{MHz}$, dipolar coupling strength $V_{12}=950 \mathrm{MHz}$, individual and collective decay rates $\Gamma_{1}=\Gamma_{2}=2 \pi \times 50$ $\mathrm{MHz}, \Gamma_{12}=2 \pi \times 9 \mathrm{MHz}$; with $\Delta_{e}=-160 \mathrm{MHz}$, for a laser coupling $\ell_{i}=200 \mathrm{MHz}$. Here, we have calculated, directly from Eq. (1), the steady state occupation probabilities for the states $|01\rangle,|10\rangle$, and $|11\rangle$ as a function of the laser detuning. We can also see from Fig. 1(a), the effect due to a different dipolar coupling, as shown by the dashed curves, for $V_{12}=50 \mathrm{MHz}$. Since this implies a change in the relative position of the coupled dipoles, this is reflected in a shift of the resonant peaks at frequencies $\nu_{1}$, and $\nu_{2}$, and a decreasing of the signal intensity coming from the two-photon emission process (intermediate peak) due to the weaker dipolar coupling.

The case of molecules with equal transition frequencies $\left(\Delta_{-}=0\right)$ is illustrated by the continuous curves in Fig. 1 (b), for $V_{12}=950 \mathrm{MHz}$. As expected, only two peaks appear: one associated to the resonance condition $\nu_{1}=\nu_{2}$, and the intermediate one, associated to the dipolar coupling. By bringing the dipoles into resonance, a clear increment in the two-photon resonance signal, in comparison to the experimentally measured spectra [22] (calculated as the dashed curve in Fig. 1(b)) is observed, thus indicating a stronger dipole-dipole coupling.

The resonance fluorescence spectrum reported in 22 can be theoretically reproduced by plotting the steady state quantity $\rho_{01,01}+\rho_{10,10}+2 \rho_{11,11}$, as shown in Fig. 1 (c). This shows the appearance of the two-photon resonance as the laser excitation intensity is increased. This peak is shifted from the laser detuning $\Delta_{+}=0$ due to the 

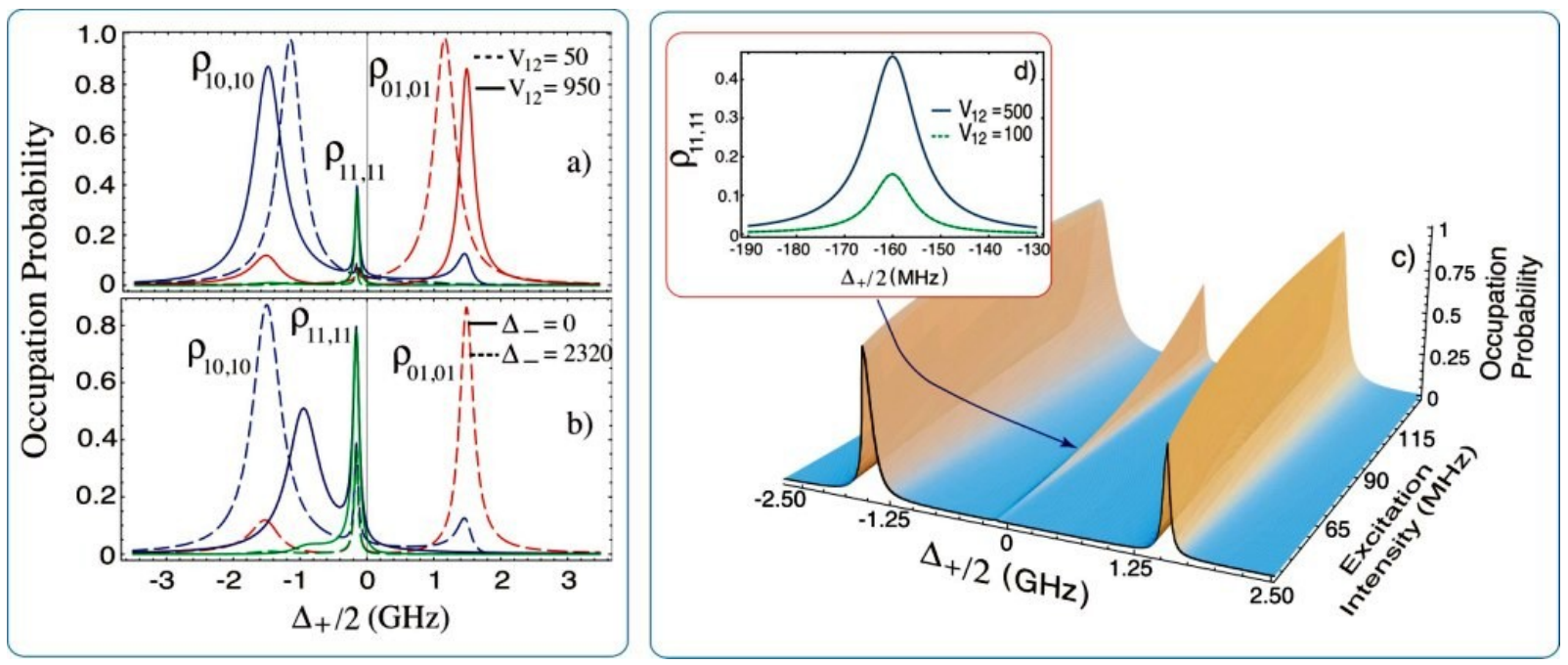

FIG. 1: Steady state occupation probabilities for the dimer system. The detunings $\Delta_{-} \equiv \nu_{1}-\nu_{2}$, and $\Delta_{+} / 2 \equiv\left(\nu_{1}+\nu_{2}\right) / 2-\nu_{L}$. a) Populations $\rho_{i j, i j}$ for two different dipolar couplings, $\Delta_{-}=2320 \mathrm{MHz}$. b) Probabilities for a fixed dipolar coupling, $=950$ $\mathrm{MHz}$, and different molecular resonance conditions. In both cases, $\Gamma_{1}=\Gamma_{2}=2 \pi \times 50 \mathrm{MHz}, \Gamma_{12}=2 \pi \times 9 \mathrm{MHz}, \Delta_{e}=-160 \mathrm{MHz}$ and $\ell_{i}=200 \mathrm{MHz}$. c) Fluorescence spectrum given in terms of the occupation probabilities, as a function of the laser coupling strength and the laser detuning $\Delta_{+} / 2$. In MHz, $\Gamma_{i}=18 \pi, \Gamma_{12}=9 \pi, \Delta_{-}=2320$, and $\Delta_{e}=-160$. d) Zoom of the two photon (intermediate) resonance for $\ell_{i}=100 \mathrm{MHz}$.

the vacuum fluctuation noise, an effective shift that has been experimentally [22] and theoretically [23] estimated at around $-160 \mathrm{MHz}$. The calculations here presented have been done for the independent and collective decay rates $\Gamma_{i}=18 \pi \mathrm{MHz}, \Gamma_{12}=9 \pi \mathrm{MHz}$. Figure 1 $(\mathrm{d})$ shows a zoom of the intermediate resonance of Fig. 11(c), for a laser coupling $\ell_{i}=100 \mathrm{MHz}$, and different dipolar couplings. This shows a clear increment in the size of the resonance as the qubit coupling is increased. If this graph were to be calculated for $\ell_{i}=10 \mathrm{MHz}$ (not shown), the probability of simultaneous excitation $\rho_{11,11}$ appears multiplied by the scale factor $10^{-5}$. Hence, the resonance signal can be tailored according to designed structural molecular parameters. The difference in the half-width of the reported peaks has its origin in the constructive and destructive interference of the different possible relaxation pathways [25].

\section{DIMER ENTANGLEMENT EVOLUTION}

As a norm for the entanglement quantification of the dipole-coupled molecules, we use Wootter's concurrence $C(\rho)=\max \left\{0, \lambda_{1}-\lambda_{2}-\lambda_{3}-\lambda_{4}\right\}$, where the $\lambda_{i}$ 's are the square roots of the eigenvalues of the non-hermitian matrix $\rho \widetilde{\rho}$, arranged in decreasing order, with $\widetilde{\rho}=\sigma_{y}^{(1)} \otimes$ $\sigma_{y}^{(2)} \rho^{*} \sigma_{y}^{(1)} \otimes \sigma_{y}^{(2)}[26]$. We consider the initial state density matrix

$$
\rho(0)=\left(\begin{array}{cccc}
a & 0 & 0 & w \\
0 & b & z & 0 \\
0 & z^{*} & c & 0 \\
w^{*} & 0 & 0 & d
\end{array}\right)
$$

as this allows the initialization of a broad class of entangled states [11, 14. In the standard two-qubit computational basis, the maximally entangled Bell state $\left|\psi^{+}\right\rangle=\frac{1}{\sqrt{2}}(|01\rangle+|10\rangle)$ is obtained for $a=w=d=0$; $b=c=z=1 / 2$. All the other states of the Bell basis, as well as the Werner mixed state [14] can be produced in a similar manner.

\section{A. Coherent control dynamics}

We consider the dynamics of the initial entangled state $\left|\psi_{0}(\alpha)\right\rangle=\sqrt{\alpha}|01\rangle+\sqrt{\beta}|10\rangle$, where $\alpha$ is a real number, $\sqrt{\beta}=|\sqrt{\beta}| e^{i \phi}$ is a complex number of phase $\phi$, and $\alpha+|\beta|=1$. This choice follows the identification of the eigenstates of the system's Hamiltonian $\hat{H}_{S}$, in the absence of external driving. If no cooperative phenomena due to $V_{12}$ (decay rate $\Gamma_{12}$ ) take place (for example, due to a long separation between the molecules, $V_{12} \sim 0$ ), in the absence of laser driving, the entanglement of this type of state has a natural asymptotic time decay due to the emission rates $\Gamma_{i i} \equiv \Gamma_{i}$ of the individual molecules.

The numerical solution of the master equation (1) allows the quantification of the dimer entanglement dynamics, as shown in Fig. 2, where the external field has been used to produce a coherent control of entanglement. Figure 2(a) shows that for a strong laser coupling $\left(\ell_{i}=500 \mathrm{MHz}\right)$ the concurrence abruptly decays to zero for a time less than $t_{\Gamma}$, where $t_{\Gamma} \equiv 1 / \Gamma$ is the time associated to the decay rates $\Gamma_{i} \equiv \Gamma$ for all the initial state configurations (all $\alpha$ ). In this work, $\Delta_{+}, \Delta_{-}, \Delta_{e}, V_{12}$, and $\ell_{i}$ are given in frequency $(\nu \equiv \omega / 2 \pi)$ units, whereas and $\Gamma_{i j}$ appear divided by a $2 \pi$-factor to consistently give 

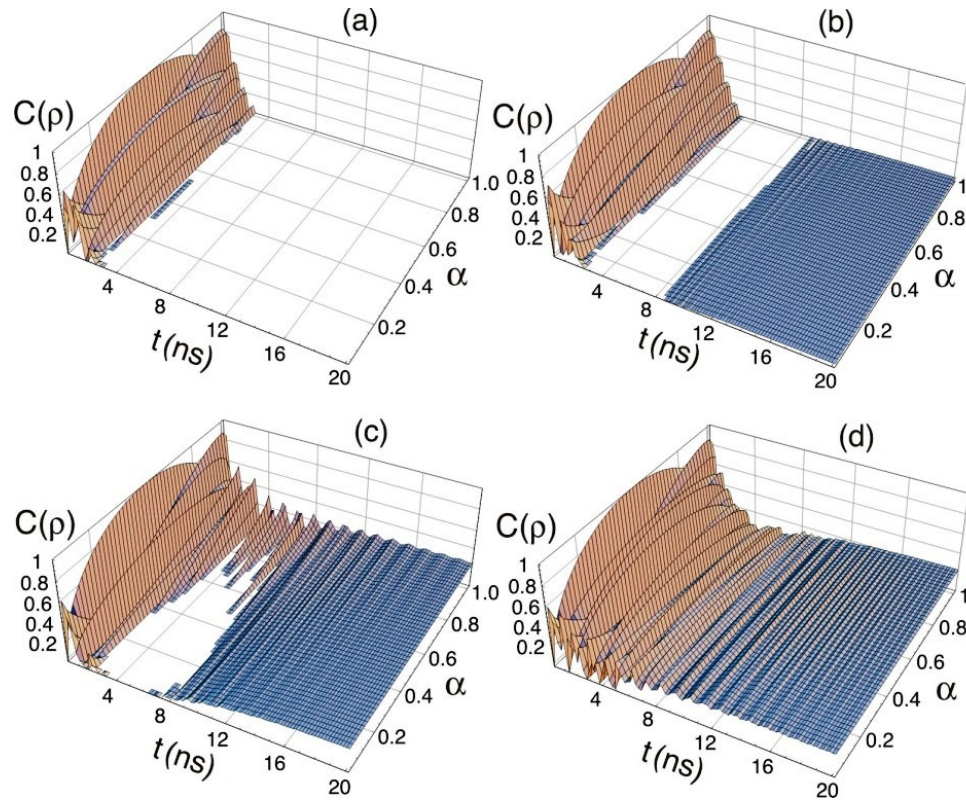

FIG. 2: Laser-driven entanglement dynamics control. a) Early stage disentanglement: $\Delta_{+}=0$, and $\ell_{1}=\ell_{2}=$ $500 \mathrm{MHz}$. b) The molecules couple to the laser with different strengths: $\ell_{1}=300 \mathrm{MHz}$, and $\ell_{2}=500 \mathrm{MHz}$. Figures c) $\Delta_{+}=\Delta_{-}$, and d) $\Delta_{+}=-\Delta_{-}$, show the difference in entanglement behavior when the laser resonant condition is varied, for $\ell_{1}=300 \mathrm{MHz}, \ell_{2}=500 \mathrm{MHz} . \Delta_{e}=0$. In all the graphs, $\Gamma_{1}=\Gamma_{2}=2 \pi \times 50 \mathrm{MHz}, \Gamma_{12}=2 \pi \times 9 \mathrm{MHz}, \Delta_{-}=2320 \mathrm{MHz}$, and $V_{12}=950 \mathrm{MHz}$.

the same units. This early stage disentanglement is due to the vacuum fluctuations affecting each molecule separately, and radically changes when the coupling between the laser and the molecules $\ell_{1} \neq \ell_{2}$, as shown in Fig. 2(b). There, for all $\alpha$, a finite, stationary entanglement $C(\rho)$ remains for long times. This behavior occurs af-C ter a period for which the system experiences a complete disentanglement $-C(\rho)=0$ for all $\alpha$, and can be further controlled, as shown in Figs. 2(c) and (d), by tuning the molecule resonance conditions with the laser field.

In Fig. 2(c), the ESD behavior is partially suppressed for finite $\alpha(0.8 \lesssim \alpha \leq 1)$, when the laser is on resonance with the qubit $2\left(\nu_{L}=\nu_{2}\right)$, that is, for $\Delta_{+}=\Delta_{-}$ $\left(\nu_{1}>\nu_{2}\right)$. In this case, the asymptotic limit of the concurrence is increased to $\sim 0.25$. In Fig 2 (d), the frequency of the external field has been tuned to be in resonance with that of qubit $1\left(\Delta_{+}=-\Delta_{-}\right)$: the ESD behavior is now completely suppressed for all $\alpha$, and the entanglement shows an oscillatory decay. For long times, and for all $\alpha$, the concurrence approaches the same stationary entanglement as before. The fast oscillations of the concurrence are due to the competition between both the dipolar coupling and the laser-molecule interactions. Thus, the results of Figs. 2(c) and (d) give a mechanism for coherent control of the dimer's ESD behavior, and for the generation of steady-state entanglement.

Figure 3 shows another interesting behavior in the con- currence dynamics-the sudden birth of entanglement. In these graphs, the system is prepared in the initial separable state $\left|1_{1}\right\rangle \otimes\left(\sqrt{\alpha}\left|0_{2}\right\rangle+\sqrt{\beta}\left|1_{2}\right\rangle\right)$, and after a certain time, which depends on the type of input superposition (i.e., on the value of $\alpha$ ), entanglement appears in the system (see Figs. 3(a), and (c)). Note that, due to the detuning between the molecular frequencies, the different resonant laser tuning of the molecules produces different entanglement behavior.

Figure 3(a) shows a delayed ESB for $\alpha=0$ and $\alpha$ values close to zero. The fast oscillations of the concurrence in Fig. 3(a) are plotted in its inset for about $1 / 16$ of the total time-frame considered in the main figure. The graph 3(c) shows the effect due to the change in the laser oresonance molecular tuning. Here, $\Delta_{+}=-\Delta_{-}$, and an appreciable ' $\alpha$-region' of early stage disentanglement is suppressed, accompanied by an increment in the amount of generated stationary entanglement. As a major feature, we notice that due to the laser addressing strategy (let's recall that the two dipoles have different transition energies), the initial states $|0\rangle \otimes|1\rangle$, and $|1\rangle \otimes|0\rangle(\alpha \equiv 1)$ are more prone to a quick generation of entangled states than the initial condition $|1\rangle \otimes|1\rangle(\alpha \equiv 0)$; in the latter there is an initial lack of entanglement generation, and ESB behavior takes place. In the former, the quick entanglement generation can be understood from the fact that a coherent superposition of the states $|0\rangle \otimes|1\rangle$ and $|1\rangle \otimes|0\rangle$ is naturally supported by the dipole-dipole interaction; in fact, in the absence of laser tuning, such a superposition of states produce some of the eigenstates of the system's Hamiltonian. The time scale for which ESB
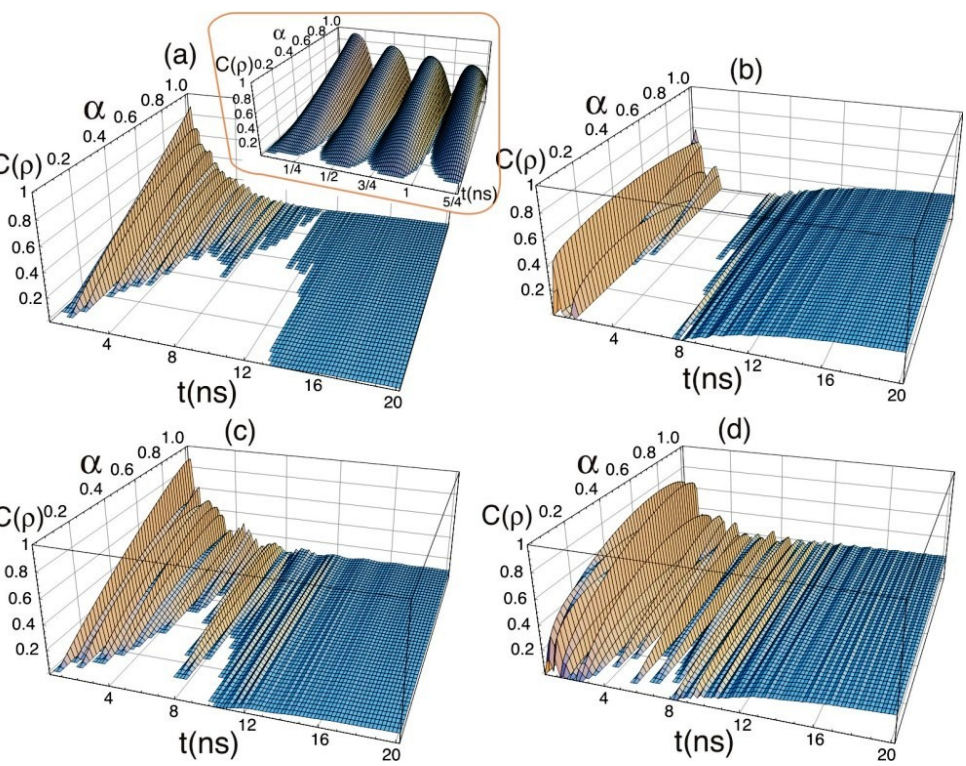

FIG. 3: Entanglement dynamics and ESB. Initial separable state $|1\rangle(\sqrt{\alpha}|0\rangle+\sqrt{\beta}|1\rangle)$, (a) $\Delta_{+}=\Delta_{-}, \ell_{1}=\ell_{2}=100 \mathrm{MHz}$; (b) $\Delta_{+}=\Delta_{-}, \ell_{1}=300 \mathrm{MHz}, \ell_{2}=500 \mathrm{MHz}$. (c) $\Delta_{+}=$ $-\Delta_{-}, \ell_{i}=100 \mathrm{MHz}$; (d) $\Delta_{+}=-\Delta_{-}, \ell_{1}=300 \mathrm{MHz}$, and $\ell_{2}=500 \mathrm{MHz} . \quad \Gamma_{1}=\Gamma_{2}=100 \pi \mathrm{MHz}, \Gamma_{12}=18 \pi \mathrm{MHz}$, $\Delta_{-}=2320 \mathrm{MHz}, \Delta_{e}=-160 \mathrm{MHz}$, and $V_{12}=950 \mathrm{MHz}$. 


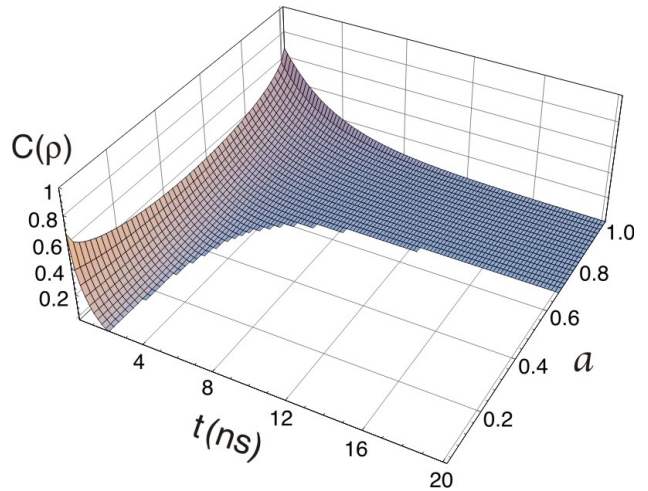

FIG. 4: Entanglement dynamics for initial entangled states given by Eq. (3), with $a \mapsto \frac{a}{3}, b=c=z=\frac{1}{3}, w=0$, and $d=\frac{1-a}{3}$; under spontaneous emission. $\Delta_{-}=2320 \mathrm{MHz}$, $\Delta_{+} / 2=\left(\nu_{1}+\nu_{2}\right) / 2 \approx 1 \times 10^{9} \mathrm{MHz}$ (optical transition). The decay rates $\Gamma_{i} \equiv \Gamma=100 \pi \mathrm{MHz}, \Gamma_{12}=0$; the remaining parameters $\left(V_{12}, \ell_{i}\right.$ and $\left.\Delta_{e}\right)$ are set to zero.

takes place depends on the qubit resonance conditions: for the initial condition $|1\rangle \otimes|1\rangle$, the ESB appearance in Fig. 3(a) takes a longer time than that calculated for Fig. 3(c).

Figures 3(b) and (d) show the effect due to increasing the laser intensity. A quicker generation of entanglement at around $\alpha \equiv 0$ is now produced (cf. Figs. 3 (a) and (c)), with a subsequent generation of ESD (Fig. $3(\mathrm{~b})$ ), and a partial ESD suppression (Fig. 3(d)), followed by an overall increment in the amount of generated stationary entanglement, $C \sim 0.2$. These graphs manifest the effect due to the tuning of the molecular resonance: if $\Delta_{+}=-\Delta_{-}$(Fig. $\left.3(\mathrm{~d})\right)$ the timeframe for the region of zero concurrence of Fig. 3(c) is almost completely suppressed, in addition of a larger number of entanglement oscillations before the steadystate is reached. For the chosen initial condition, the $\Delta_{+}=-\Delta_{-}$-resonance condition favors the entanglement production and inhibits ESD behavior. The same conclusion can be reached if we instead consider, as initial condition, the state $(\sqrt{\alpha}|0\rangle+\sqrt{\beta}|1\rangle) \otimes|1\rangle$ (not shown). The changes in the entanglement features shown in Fig. 3 are not only due to the chosen initial conditions and resonance features, but also to the fact that, in the considered scenarios, we have taken into account the effective energy shift $\Delta_{e}=-160 \mathrm{MHz}$, which is associated to the doubly excited state, as reported in [22, 23]. The numerical values for the strength of the molecular interaction, the decay rates, and the molecular transition energies used in the simulations of Fig. 2 and 3 are those reported in the experiment of Ref. [22].

\section{B. Dependence on the initial state preparation}

Further to our previous analysis, we plot in Fig. 4 the entanglement dynamics for an initial state of the form
Eq. (3), with $a \mapsto \frac{a}{3}, b=c=z=1 / 3 ; w=0$, and $d=\frac{1-a}{3}$. Due to the structure of the density matrix, the concurrence allows an analytical calculation of the observed ESD behavior, and the associated ESD time $\tilde{t}$ (for each $a$ ) can be exactly estimated. For example, for $a=0, \tilde{t}=\frac{1}{\Gamma} \ln \left|\frac{2+\sqrt{2}}{2}\right|$, which means $\tilde{t} \approx 1.7 \mathrm{~ns}<\Gamma^{-1}$ in our molecular set-up. This behavior is observed over a continuous range of $a$ values, and for any $0 \leq a<2 / 3$ is given by

$$
\tilde{t}=\frac{1}{\Gamma} \ln \left(\frac{1-a}{2-3 a}\left[2-a+\sqrt{a^{2}-a+2}\right]\right) .
$$

For $a \geq 2 / 3$, ESD is suppressed and entanglement decays in an asymptotic fashion. Hence, the dissipative molecular dynamics given by Eq. (1) exhibit a rich behavior and both an ESD dynamics and an asymptotic decay of entanglement can be induced in the dimer system by tuning the initial condition ( $a$ value).

In Fig. 5 we consider the entanglement dynamics for the general initial product state of qubit superpositions

$$
\left|\Psi_{0}(\gamma)\right\rangle=\left(\sqrt{\gamma}\left|0_{1}\right\rangle+\sqrt{\delta}\left|1_{1}\right\rangle\right) \otimes\left(\sqrt{\zeta}\left|0_{2}\right\rangle+\sqrt{\eta}\left|1_{2}\right\rangle\right),
$$

where, as before, $\gamma+|\delta|=1, \zeta+|\eta|=1$. Here, we plot the corresponding concurrence as a function of $\gamma$, for $\zeta=0,1 / 2,1(\sqrt{\zeta}>0)$. We note that different initial preparations for the disentangled type of states lead to very different entanglement behavior. For instance, Fig. 5 (a) exhibits a sudden birth of entanglement dynamics for low values of $\gamma$, whereas for their intermediate values there are collapses and revivals that eventually become suppressed as $\gamma$ is increased; from this point, coherent oscillations of entanglement persist to finally decay and then converge to a stationary value. In fact, the concurrence evolves, for all $\gamma$, towards a finite non-zero stationary value due to the inter-qubit coupling strength and the driving field. Also note that for short times the molecular entanglement reaches its maximum for $\gamma \mapsto 1$. Fig. 5(b) illustrates the case of equal superpositions in both qubits. Unlike the previous case, here there is a finite entanglement oscillation at short times for $\gamma \mapsto 0$, which can be understood in terms of the symmetry of the initial state. Here there is no presence of ESB behavior. Due to the initial condition, Fig. 5(c) shows, in contrast to Fig. 5(a), the highest entanglement oscillations for $\gamma \mapsto 0$. In all cases, the oscillations approach the same stationary value, as previously observed. Notice that in Fig. 5 we have considered a non-zero value for $V_{12}$ and for the $\Delta_{e^{-}}$ shift, in contrast to the graph of Fig. 4. The parameters used in this simulation have been taken from the experimental data of Ref. 22. We have recently learned, although in a different context, that steady state entanglement is also produced if counter-rotating wave terms are kept in the original system Hamiltonian 8, 27.

Figure 6 follows a similar analysis as before, but for the case in which there is no physical interaction between the molecules, and therefore no collective decay. We consider instead the effects due to the vacuum fluctuations 

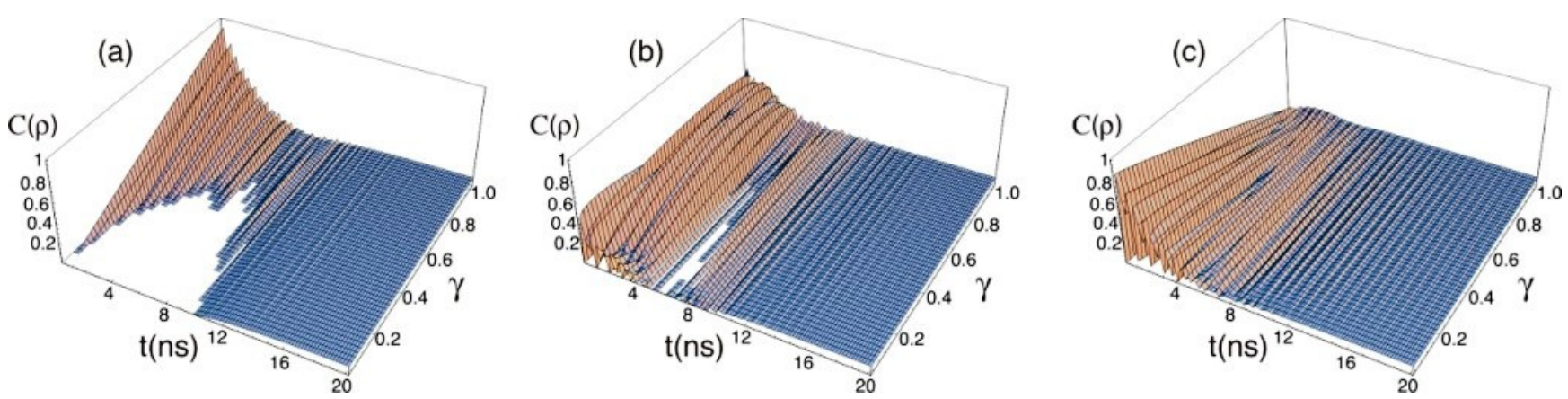

FIG. 5: Initial state preparation of product states $\left|\Psi_{0}(\gamma)\right\rangle=(\sqrt{\gamma}|0\rangle+\sqrt{\delta}|1\rangle) \otimes(\sqrt{\zeta}|0\rangle+\sqrt{\eta}|1\rangle)$, for a) $\zeta=0$, b) $\zeta=1 / 2$, c) $\zeta=1$. $\Delta_{-}=2320 \mathrm{MHz}, \Delta_{+}=0$. The decay rates $\Gamma_{i}=100 \pi \mathrm{MHz}, \Gamma_{12}=18 \pi \mathrm{MHz}$. $\ell_{i}=100 \mathrm{MHz}, \Delta_{e}=-160 \mathrm{MHz}$, and $V_{12}=950 \mathrm{MHz}$.
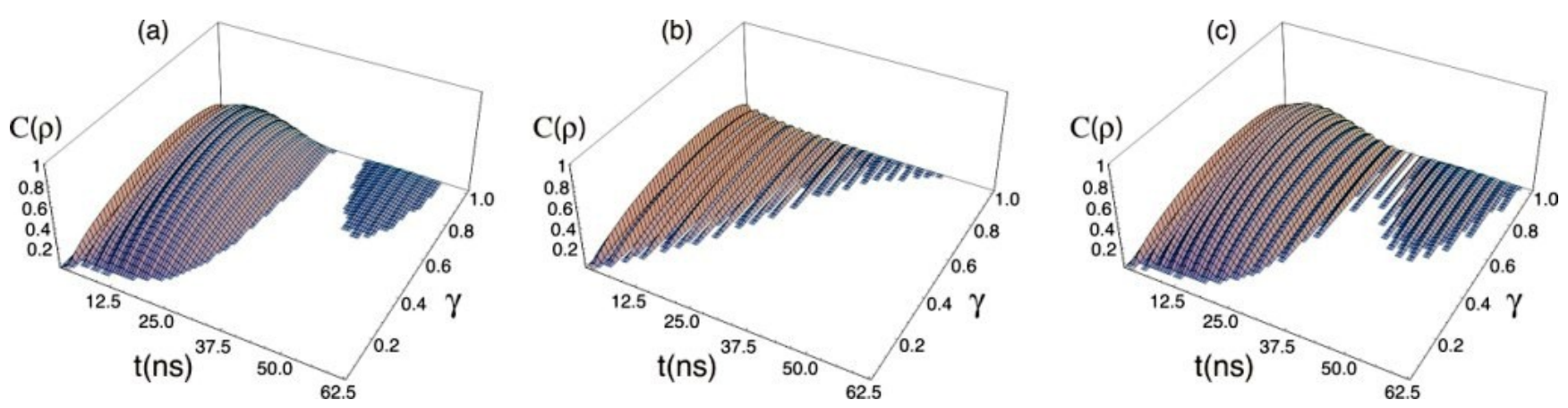

FIG. 6: Initial product states $\left|\Psi_{0}(\gamma)\right\rangle$ (Eq. (5)), in the absence of qubit-qubit interaction and collective decay $\left(V_{12}=0, \Gamma_{12}=0\right)$, for a) $\zeta=0$, b) $\zeta=1 / 2$, c) $\zeta=1 . \Delta_{-}=2320 \mathrm{MHz}, \Delta_{+}=2638 \mathrm{MHz} . \Gamma_{i}=10 \pi \mathrm{MHz}, \ell_{i}=200 \mathrm{MHz}, \Delta_{e}=-160 \mathrm{MHz}$.

$\Delta_{e}$-the Lamb shift experienced by the molecular doubly excited state, and to the external driving field. The results are clearly different from those of Fig. 5. even though the qubits start from a separable state, they entangle due to the radiative corrections and the external field. The concurrence exhibits a similar behavior for the conditions a) $\zeta=0$, and c) $\zeta=1$, for which there is a maximum amount of generated entanglement, and ESD and collapse-revival behavior are present as a function of the initial qubit preparation. The scenario $\zeta=1 / 2$ (graph 6(b)) does exhibit a different type of decay, and unlike the previous cases, there are not two "islands" of $\mathrm{C}(\rho)$ entanglement, and the oscillations persist the most for $\gamma \mapsto 1$. Notice that the time scale for such oscillations is much longer than those of the graphs previously shown.

\section{Laser coupling}

We address the entanglement effects due to the molecular coupling to the laser field, for the initial condition given by Eq. (5). Figure 7 shows the entanglement evolution as a function of the laser field coupling strength $\ell$. In the figure, $\gamma=\zeta=1 / 2$, and the other parameters are given as in Fig. 5 , for a) $V_{12}=950 \mathrm{MHz}$, and b) $V_{12}=50$ $\mathrm{MHz}$. Figure 7(a) shows that for the considered initial product state of superpositions there is an optimal laser coupling strength for which maximum stationary entanglement is generated. Away from this optimal value, the stationary entanglement decreases monotonically until it reaches a certain intensity from which any entanglement correlation is washed away and the concurrence goes to zero after a few oscillations. This indicates that the laser can be used to tune and maximize stationary entanglement production, given an initial condition. (a)

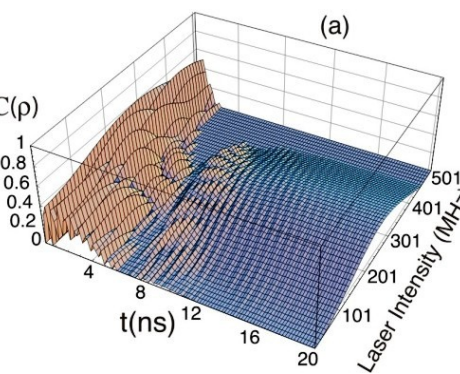

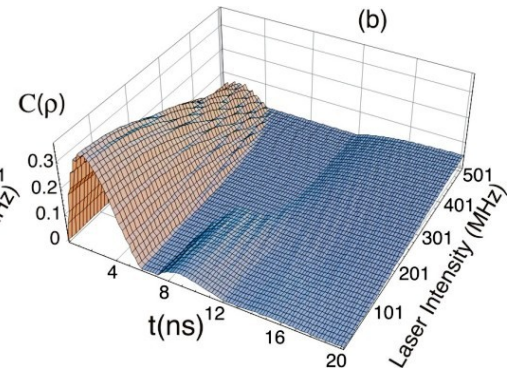

FIG. 7: Concurrence dynamics as a function of the laser intensity $\ell_{i} \equiv \ell$, for the initial condition $\left|\Psi_{0}(\gamma)\right\rangle$, with $\gamma=\zeta=1 / 2$. a) $V_{12}=950 \mathrm{MHz}$, b) $V_{12}=50 \mathrm{MHz} . \Delta_{-}=2320 \mathrm{MHz}$, $\Delta_{+}=0, \Gamma=100 \pi \mathrm{MHz}, \Gamma_{12}=18 \pi \mathrm{MHz}, \Delta_{e}=-160 \mathrm{MHz}$.

In Fig. 7(b) we have decreased $V_{12}$ to $50 \mathrm{MHz}$. In contrast to the previous case, for low laser coupling strength, there are no entanglement oscillations before the concurrence reaches its minimum value: the concurrence 
exhibits an island whose height depends on the laser strength. Unlike the previous case, here the maximum entanglement occurs at low intensity, due to the fact the qubit-qubit interaction is smaller than $\Delta_{e}$, which dominates the dynamics. The entanglement rapidly vanishes as the laser intensity increases, with the two-particle state becoming separable during a period whose length increases with the laser intensity. It then starts to build up towards a stationary degree of entanglement, for which there is also a maximum value which is directly dependent on the intensity of the laser. After this, the entanglement gradually decays as the laser intensity is further increased (not shown) and eventually vanishes following a similar pattern to that of Fig. 7(a).
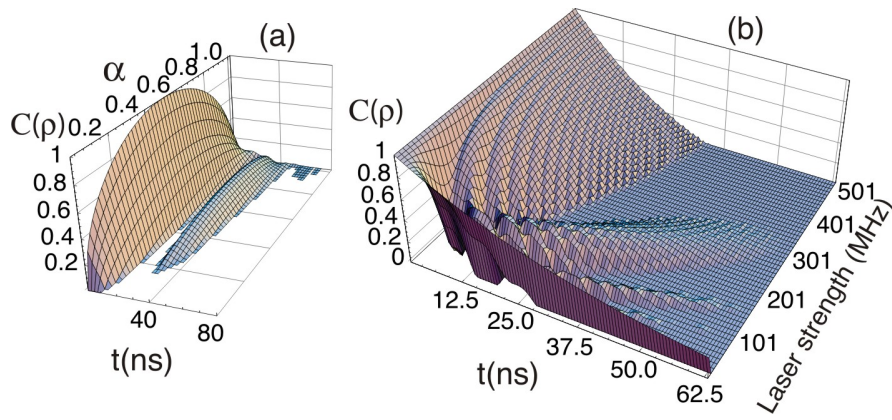

FIG. 8: Entanglement dynamics as a function of the initial condition and laser intensity, for the initial configuration $\left|\psi_{0}(\alpha)\right\rangle$. a) $\ell=200 \mathrm{MHz}$, b) $\alpha=1 / 2 . \quad \Delta_{-}=2320 \mathrm{MHz}$, $\Delta_{+}=2638 \mathrm{MHz}, \Gamma=10 \pi \mathrm{MHz}, \Delta_{e}=-160 \mathrm{MHz} . V_{12}=$ $\Gamma_{12}=0$.

For the sake of completeness, we next consider the situation of non-interacting qubits $\left(V_{12}=0\right)$, and no collective decay for the initial state $\left|\psi_{0}(\alpha)\right\rangle=\sqrt{\alpha}|01\rangle+\sqrt{\beta}|10\rangle$. Figure 8 (a) shows that ESD and collapse-revival behavior take place in the entanglement evolution as the initial condition is varied, by means of tuning $\alpha$ (e.g., maximal entanglement is attained for $\alpha=1 / 2)$. In contrast, Fig. 8(b) shows the dependence of entanglement on the laser intensity for the same initial state $\alpha=1 / 2$. Here, we distinguish three main dynamical scenarios. For low intensity values, there is an asymptotic decay of concurrence. As the intensity increases, collapse-revivals occur whereas for larger values of field coupling, pure ESD phenomena take over the dynamics. The time scale plotted in Fig. 8 is to be compared with the 'natural' decay time $t_{\Gamma} \equiv \Gamma^{-1}$. It is clearly shown that coherent laser driving can be used as a key ingredient for determining the major features of entanglement dynamics.

\section{Nonlocal fluctuations: Lamb shift correction}

We illustrate in Fig. 9 a major point of this work: in the scenario where the molecules are not subjected to the collective damping due to the vacuum field, dark periods and revival of entanglement appear. This result is in contrast to the revival of entanglement reported in [30, where such a behavior is critically dependent on the collective decay. As is shown in our case, the revival phenomenon is strictly induced by the external laser control and the quantum noise fluctuations given by the Lamb shift 23 .

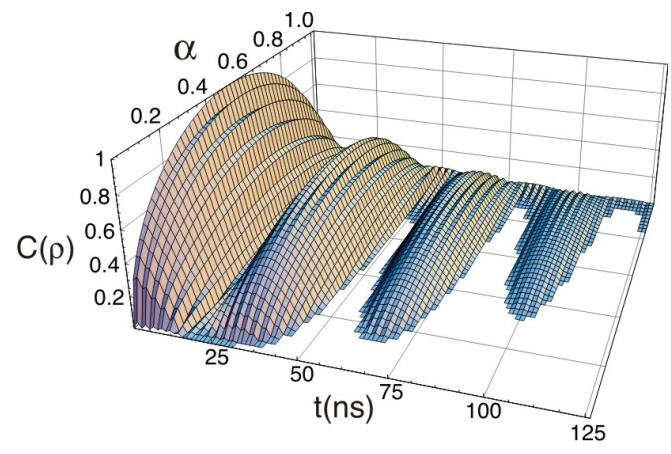

FIG. 9: Concurrence evolution as a function of the initial condition $\left|\psi_{0}(\alpha)\right\rangle$. The qubits are far apart from each other and do not experience a collective decay $\left(V_{12}=0, \Gamma_{12}=0\right)$. They, however, due to the vacuum noise, experience $\Delta_{e}=$ $-160 \mathrm{MHz}$ [22]. $\Gamma_{i}=4 \pi \mathrm{MHz}, \Delta_{-}=2320 \mathrm{MHz}, \Delta_{+}=$ $2638 \mathrm{MHz}$, and $\ell_{i}=200 \mathrm{MHz}$.

The entanglement revival behavior occurs even when the pair of molecules do not interact, just as a consequence of the noise due to vacuum electromagnetic fluctuations. Such fluctuations are always present [28, and the molecules experience a vacuum noise input and radiate a vacuum noise output. This effect shifts the energy levels of the molecules 29. The so-called Lamb shift has been calculated for a specific molecular system in [23. We show such an effect in Fig. 9, where we have considered a qubit separation such that $V_{12}=0$, and $\Gamma_{12}=0$. We have introduced a phenomenological $\Delta_{e}$-shift, starting from the experimental results reported in [22], and the calculations of Ref. 23 .

Our result is also in contrast with that obtained in Ref. 15. There, the authors attribute the revival behavior (similar to ours) to the memory (non-Markovian) effects induced by two reservoirs acting on the qubits in an independent manner. Figure 9 shows that the revival of entanglement is not, strictly speaking, a nonMarkovian feature associated to an uncoupled two-qubit system. This shows that for the case of independent spontaneous emission decay, a similar behavior to that reported in [15] can be found. Our result, however, has been derived within a Born-Markov description, and as such, does not invoke a non-Markovian treatment. On the same grounds, this is different from the collapserevival behavior reported for the interacting system of Ref. 31. The behavior showed in Fig. 9 changes with increasing emission rates (cf. Fig. 8): when the system decays more rapidly, the collapse-revival behavior disappears. On the other hand, Fig. 8(b) clearly shows that, for a fixed rate, collapse-revivals are suppressed for higher values of laser intensity, for which ESD becomes 
the relevant dynamical decay.

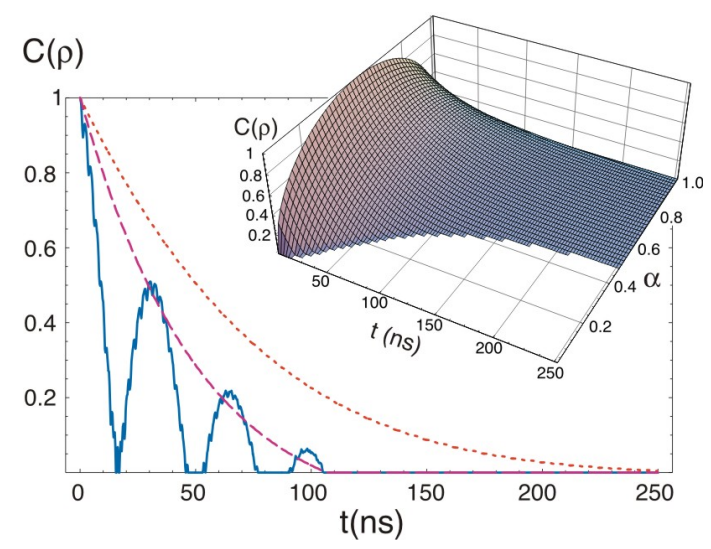

FIG. 10: Comparison between the effects due to the laser and the vacuum fluctuations over the entanglement dynamics. In the main graph, the solid curve exhibits collapses and revivals; $\ell_{i}=200 \mathrm{MHz}, \Delta_{e}=-160 \mathrm{MHz}$. The long dashed curve exhibits ESD; $\ell_{i}=200 \mathrm{MHz}, \Delta_{e}=0$. The short dashed curve corresponds to $\ell_{i}=1 \mathrm{MHz}$, and $\Delta_{e}=-160 \mathrm{MHz}$. The initial state is $\left|\psi_{0}(1 / 2)\right\rangle$. The remaining parameters used in the simulations are the same as in Fig. 9 By switching to the initial state $\sqrt{\alpha}|00\rangle+\sqrt{\beta}|11\rangle$, the inset shows the ESD sensitivity to the initial condition; $\Delta_{+}=20000 \mathrm{MHz}, \Delta_{e}=$ $-160 \mathrm{MHz}$, and $\ell_{i}=0$; the other parameters are as in Fig. 9

We have plotted, in Fig. 10, the dynamics of the concurrence for the initial entangled state $\left|\psi_{0}(\alpha=1 / 2)\right\rangle$, to show the interplay between the laser driving and the shift experienced by the doubly excited state due to background noise fluctuations. The concurrence decays asymptotically when the laser coupling is absent, in agreement with our previous results.

The main graph of Fig. 10 also shows sudden disentanglement (but not revival) when the laser coupling is strong and there is no contribution arising from the vacuum noise (long dashed curve). This behavior is also observed in the inset of Fig. 10, for a different initial condition, $\sqrt{\alpha}|00\rangle+\sqrt{\beta}|11\rangle$ : the ESD is more pronounced when $\alpha$ tends to zero, and it vanishes for $\alpha>1 / 2$. This is because in our density matrix configuration, $\rho_{00,00}$ corresponds to the lowest system eigenstate in the standard basis, and hence, for $\alpha \rightarrow 1$, the system has a higher probability of decaying to the ground state and the interesting quantum effects are lost.

The sensitivity of the ESD dynamics to the initial conditions is shown in the inset of Fig. 10. In the main figure, the entanglement decays asymptotically (uppermost curve, $\ell=1$ ) for the initial conditions $\sqrt{\alpha}|01\rangle+\sqrt{\beta}|10\rangle$ (for all $\alpha$ ), and hence ESD never takes place. However, the inset of Fig. 10 shows that for the initial condition $\sqrt{\alpha}|00\rangle+\sqrt{\beta}|11\rangle$, and absence of laser driving, the entanglement decays abruptly for values of $\alpha$ close to zero, and hence ESD is reinforced at very early times $\tilde{t}$. This clearly indicates that different types of initial entangled states experience different input noise from the environment.
An analytical expression for the concurrence, for the molecular input values of the inset of Fig. 10, follows from the calculation of the non trivial density matrix elements found for the solution of the master equation:

$$
\begin{aligned}
& \rho_{00,00}=1+(1-\alpha) \mathrm{e}^{-2 \Gamma t}\left(1-2 \mathrm{e}^{\Gamma t}\right), \\
& \rho_{01,01}=\rho_{10,10}=(1-\alpha) \mathrm{e}^{-2 \Gamma t}\left(-1+\mathrm{e}^{\Gamma t}\right), \\
& \rho_{11,11}=(1-\alpha) \mathrm{e}^{-2 \Gamma t}, \\
& \rho_{00,11}=\rho_{11,00}^{*}=\sqrt{(1-\alpha) \alpha} \mathrm{e}^{\mathrm{i} t\left(2 \pi \Delta_{e}+\mathrm{i} \Gamma+4 \pi \Delta_{+}\right)} .
\end{aligned}
$$

The concurrence $C(\rho)=2 \max \left\{0,\left|\rho_{00,11}\right|-\rho_{01,01}\right\}$. Thus, the ESD time can be directly obtained from the condition $\left|\rho_{00,11}\right|-\rho_{01,01} \leq 0$; for any $\alpha<1 / 2$, this reads

$$
\tilde{t}_{\alpha}=\frac{1}{\Gamma} \ln \left|\frac{1-\alpha}{1-\alpha-\sqrt{\alpha-\alpha^{2}}}\right| .
$$

For $\alpha \geq 1 / 2$ there exists no solution with physical meaning for $\tilde{t}_{\alpha}$, in agreement with what is shown in the inset of Fig. 10. An experimental study on the engineering and modification of the molecules decay rates has already been reported 32] this could facilitate the observation of the effects reported in Figs. 9 and 10.

\section{CONCLUSIONS}

We summarize the major findings of the present work on dimer entanglement dynamics. We have shown how the entanglement evolution, which includes ESD, ESB, and collapse-revival dynamics, can be precisely controlled by means of an external driving field, and an appropriate qubit initialization. In addition, the light-matter interaction can be used to produce transitions between the system states in such a way that a reliable production of steady state entanglement is favored. There are entanglement dynamics corrections due to the radiative shift of the doubly-excited molecular state arising from the vacuum fluctuations: in the scenario where the qubits do not experience collective coupling effects (only independent interaction with the environment), the entanglement exhibits a collapse-revival behavior. On the other hand, by considering different type of initial conditions, an ESD dynamics can also be induced due to the energy shift. We have shown that it is crucial, for a correct characterization of entanglement dynamics, to take into account the radiative corrections induced by the electromagnetic vacuum field, as such shifts or energy corrections permanently affect the evolution of entanglement.

\section{ACKNOWLEDGEMENTS}

This work was partially supported by Colciencias Grant 1106-452-21296, and the DAAD-Colciencias exchange (PROCOL) program. We acknowledge the hospitality of the Freiburg Institute for Advanced Studies, and the Max Planck Institute for the Science of 
Light Erlangen, where parts of this work were completed. JHR is grateful to the DAAD for a Research Stay
Award (A/09/0851). We would like to acknowledge L. L. Sánchez-Soto for a critical reading of the manuscript.
[1] N. D. Mermin, Phys. Today 38 (4), 38 (1985).

[2] C. Bennett and D. DiVincenzo, Nature 404, 247 (2000).

[3] C. H. Bennett et al., Phys. Rev. Lett. 70, 1895 (1993).

[4] N. Gisin et al., Rev. Mod. Phys 74, 145 (2002).

[5] T. Yu and J. H. Eberly, Phys. Rev. B. 68, 165322 (2003).

[6] T. Konrad et al., Nature Physics 4, 99 (2008).

[7] L. Quiroga et al., Phys. Rev. A 75, 032308 (2007).

[8] M. Scala et al., preprint arXiv:0907.5345

[9] P. J. Dodd and J. J. Halliwell, Phys. Rev. A. 69, 052105 (2004).

[10] F. Mintert et al., Phys. Rep. 415, 4, 207 (2005).

[11] T. Yu and J. H. Eberly, Phys. Rev. Lett. 93, 140404 (2004); ibid. 97, 140403 (2006).

[12] Z. Ficek and R. Tanas, Phys. Rev. A. 77, 054301 (2008).

[13] M. P. Almeida et al., Science 579, 316 (2007).

[14] T. Yu and J. H. Eberly, Science 323, 598 (2009).

[15] B. Bellomo et al., Phys. Rev. Lett. 99, 160502 (2007).

[16] M. Thorwart et al., Chem. Phys. Lett. 478, 234 (2009).

[17] F. F. Fanchini et al., preprint arXiv:0911.1096

[18] H. Ollivier and W. H. Zurek, Phys. Rev. Lett. 88, 017901 (2001).

[19] K. Hammerer et al., Phys. Rev. A 66, 062321 (2002).

[20] J. Eckel, J. H. Reina, and M. Thorwart, New J. Phys. 11, 085001 (2009)

[21] B. Lovett et al., Phys. Rev. B 68, 205319 (2003); S. C. Benjamin, B. Lovett, and J. H. Reina, Phys. Rev. A 70,
060305(R) (2004).

[22] C. Hettich et al., Science 298, 385 (2002).

[23] J. H. Reina et al., Phys. Rev. Lett. 93, 250501 (2004).

[24] G. S. Agarwal, Quantum Statistical Theories of Spontaneous Emission and their Relations to Other Approaches, Springer Tracts in Modern Physics, Vol. 70, ed. G. Höhler, Springer-Verlag, Berlin (1974).

[25] Z. Ficek and S. Swain, Quantum Interference and Coherence: Theory and Experiments, Springer Series in Optical Science, Springer (2005).

[26] W. K. Wootters, Phys. Rev. Lett. 80, 2245 (1998).

[27] L. L. Sánchez-Soto, private communication.

[28] R. Alicki et al., Phys. Rev. A 65, 062101 (2002).

[29] C. W. Gardiner and P. Zoller, Quantum Noise, Springer Series in Synergetics, Springer (2004).

[30] Z. Ficek and R. Tanas, Phys. Rev. A 74, 024304 (2006).

[31] S. Das and G. S. Agarwal, J. Phys. B: At. Mol. Opt. Phys. 42, 141003 (2009).

[32] B. C. Buchler et al., Phys. Rev. Lett. 95, 063003 (2005).

[33] In general, the expressions for the d-d interaction strength and the incoherent decay rate $\Gamma_{12}$ are given by $V_{12}=\mathcal{A}_{12}\left\{-\left[\hat{\boldsymbol{\mu}}_{1} \cdot \hat{\boldsymbol{\mu}}_{2}-\left(\hat{\boldsymbol{\mu}}_{1} \cdot \hat{\mathbf{r}}_{12}\right)\left(\hat{\boldsymbol{\mu}}_{2} \cdot \hat{\mathbf{r}}_{12}\right)\right] \frac{\cos z}{z}+\right.$ $\left.\left[\hat{\boldsymbol{\mu}}_{1} \cdot \hat{\boldsymbol{\mu}}_{2}-3\left(\hat{\boldsymbol{\mu}}_{1} \cdot \hat{\mathbf{r}}_{12}\right)\left(\hat{\boldsymbol{\mu}}_{2} \cdot \hat{\mathbf{r}}_{12}\right)\right]\left[\frac{\cos z}{z^{3}}+\frac{\sin z}{z^{2}}\right]\right\}$, and $\Gamma_{12}=$ $\mathcal{A}_{12}\left\{\left[\hat{\boldsymbol{\mu}}_{1} \cdot \hat{\boldsymbol{\mu}}_{2}-\left(\hat{\boldsymbol{\mu}}_{1} \cdot \hat{\mathbf{r}}_{12}\right)\left(\hat{\boldsymbol{\mu}}_{2} \cdot \hat{\mathbf{r}}_{12}\right)\right] \frac{\sin z}{z}+\left[\hat{\boldsymbol{\mu}}_{1} \cdot \hat{\boldsymbol{\mu}}_{2}-3\left(\hat{\boldsymbol{\mu}}_{1}\right.\right.\right.$. $\left.\left.\left.\hat{\mathbf{r}}_{12}\right)\left(\hat{\boldsymbol{\mu}}_{2} \cdot \hat{\mathbf{r}}_{12}\right)\right]\left[\frac{\cos z}{z^{2}}-\frac{\sin z}{z^{3}}\right]\right\}$, where $\mathcal{A}_{12} \equiv \frac{3 \sqrt{\Gamma_{1} \Gamma_{2}}}{8 \pi}$. 